\title{
Hemorrhagic Reversible Cerebral Vasoconstriction Syndrome: Clinical Presentation, Differential Diagnosis, Mechanism, and Treatment
}

\author{
Se-Heum Park, Sang-Hoon Lee, Kyung-Jae Park, Shin-Hyuk Kang, Dong-Hyuk Park \\ Department of Neurosurgery, Korea University Medical Center, Anam Hospital, Korea
}

Received: April 28, 2021

Accepted: April 28, 2021

Corresponding Author:

Dong-Hyuk Park, MD, PhD

Department of Neurosurgery,

College of Medicine, Korea

University Anam Hospital, 73,

Goryeodae-ro, Seongbuk-gu,

Seoul 02841, Korea

Tel: $+82-2-920-6833$

Fax: +82-2-929-0629

E-mail: doctorns@korea.com

\begin{abstract}
Reversible cerebral vasoconstriction syndrome (RCVS) is a clinical and radiologic syndrome that presents with a severe headache associated with multifocal segmental vasoconstriction of the cerebral arteries. The pathophysiology of RCVS is unknown, but changes in arterial tone seem to a key mechanism. The clinical course of RCVS is self-limiting, resolving spontaneously. RCVS usually follows a benign course, but the prognosis can vary because delayed intracerebral hemorrhage, ischemic, or hemorrhagic stroke can occur. Computed tomography angiography and digital subtraction angiography are the diagnostic methods of choice. The calcium channel blocker and analgesic agent, nimodipine, seems to reduce the severity of the headaches. This case report characterized the clinical presentation, diagnosis, differential diagnosis, clinical course, and management of RCVS to assist with differentiating it from other conditions it may be confused with.
\end{abstract}

Keywords: Reversible cerebral vasoconstriction syndrome; Primary reversible cerebral vasoconstriction syndrome; primary angiitis of the central nervous system

\section{INTRODUCTION}

Reversible cerebral vasoconstriction syndrome (RCVS) is typically characterized by clinically severe headache, neurologic deficits caused by ischemia or intracerebral hemorrhage, and radiologically observed multifocal cerebral artery vasoconstriction ${ }^{1,2)}$.

The purpose of this case report was to report the clinical treatment and management, as well as discuss the imaging characteristics, of an RCVS patient.

\section{CASE REPORT}

A 28-year-old woman with no past medical history presented with a severe headache (VAS 7) and vomiting. She underwent a cesarean section a week earlier and had no particular postoperative complications. When she came to our emergency room, her initial mental status was drowsy, and her blood pressure was normal. Computed tomography (CT) showed a left basal ganglia Intracerebral hemorrhage ( $\mathrm{ICH}$ ) and a subarachnoid hemorrhage (SAH) in the left frontal sulci (Fig. 1). Rheumatoid factor, antinuclear antibody, and inflammation-related laboratory tests were performed, all of which were normal. She complained of anxiety and dizziness. High blood pressure $(160 / 90 \mathrm{mmHg}$ ) was noted and the follow-up CT showed left intracerebral hemorrhage resolution and marked low density in the left ACA-MCA border zone (Fig. 2) on day 10. There was no difference in consciousness, and dizziness re-

Copyright (C) 2021 The Korean Neurointensive Care Society

This is an Open Access article distributed under the terms of the Creative Commons Attribution Non-Commercial License (http://creativecommons.org/licenses/by-nc/4.0/) which permits unrestricted non-commercial use, distribution, and reproduction in any medium, provided the original work is properly cited. 

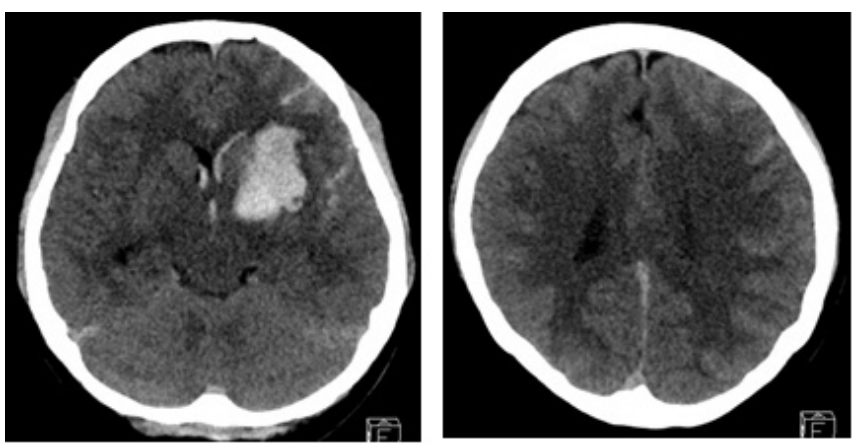

Fig. 1. Cerebral computed tomography scan on day 1 after the occurrence of mental changes (drowsy) and ICH in the Lt. basal ganglia, SAH along the Lt. cerebral sulci and IVH. ICH, intracerebral hemorrhage; Lt., left; $\mathrm{SAH}$, subarachnoid hemorrhage; IVH, intraventricular hemorrhage.
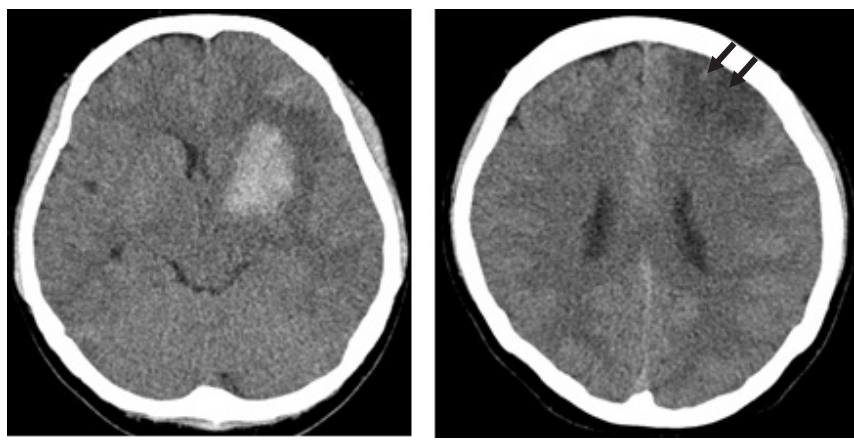

Fig. 2. Cerebral computed tomography scan on day 10 (left) and resolving process of the $\mathrm{ICH}$ in the Lt. basal ganglia, $\mathrm{SAH}$, and marked low-density in the Lt. ACA-MCA watershed zone (right, arrows).

peatedly improved and worsened. CT angiography (CTA) revealed smooth, contoured, narrowing bilateral intracranial arteries, and no demonstrable cerebral aneurysm or vascular malformation (Fig. 3). A tentative diagnosis of RCVS was made. The patient was started on nimodipine $270 \mathrm{mg}$ oral administration per day and was treated for high blood pressure. Digital subtraction angiography (DSA) was done on day 11. Diffuse vasospasm involving bilateral intracranial arteries, intraarterial drug infusion angioplasty in bilateral ICAs using nimodipine $3 \mathrm{mg}$ shows poor response of drug infusion angioplasty (Fig. 4). Magnetic resonance (MR) perfusion was performed on day 18 . No dealyments were found in the time to peak or mean transit time. The MRI T1 contrast image revealed probable ICH in the left basal ganglia, gyral enhancement along the left frontal convexity, and probable subacute infarction (Fig. 5). Her consciousness, anxiety, and dizziness improved on day 28 , without neurologic deficits.

Repeat CTA three months post-admission revealed bilateral, multifocal, reversible vasoconstriction, confirming a diagnosis of RCVS. The patient is still undergoing outpatient follow-up without any special neurologic disorders.
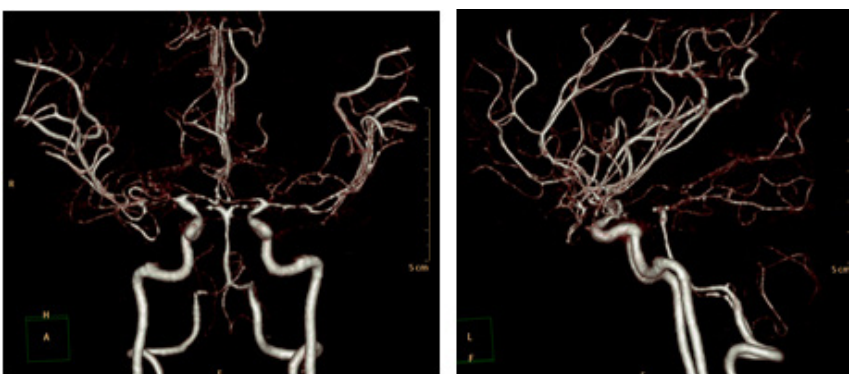

Fig. 3. CT angiography on day 10 and smooth, contoured, narrowing bilateral intracranial arteries, with no demonstrable cerebral aneurysm or vascular malformation.
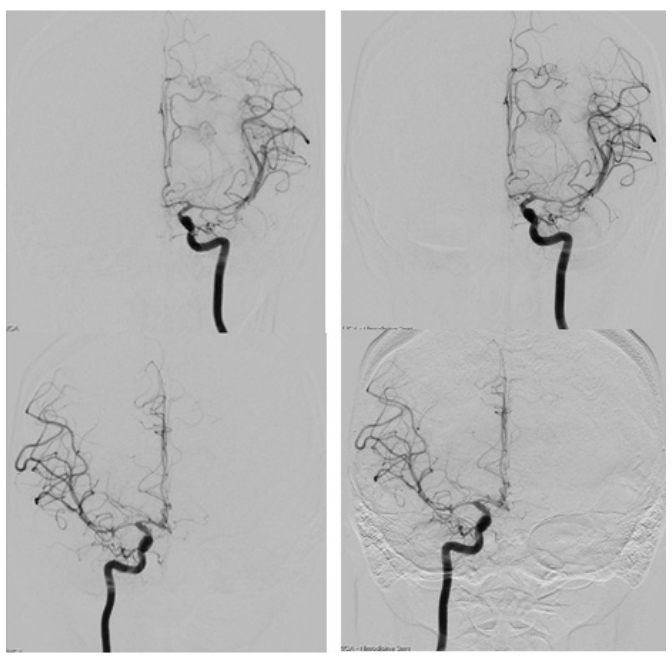

Fig. 4. DSA on day 11. Diffuse vasospasm involving bilateral intracranial arteries (left) and intraarterial drug infusion angioplasty in bilateral ICAs using nimodipine $3 \mathrm{mg}$, respectively, with poor response in drug infusion angioplasty (right).

\section{DISCUSSION}

RCVS has a clinical presentation of thunderclap headache, nausea, vomiting, photophobia, encephalopathy, seizure, focal neurologic deficit due to ischemia, cerebral edema, and cerebral infarc$\operatorname{tion}^{3)}$. An initial DSA and CTA are the preferred imaging modalities, and such images show multifocal segmental vasoconstriction and reversibility of angiographic spasms in the three months following the onset of clinical symptoms ${ }^{4}$. Although the pathophysiology is still not yet clearly defined, multiple factors can cause $\mathrm{SAH}$ including pial vessels and reperfusion injury due to high flow in spastic vessels ${ }^{3}$. Sympathetic hyperreactivity by medication, endothelial dysfunction, and oxidative stress are prone to generating vasoconstriction, causing $\mathrm{RCVS}^{4}$. The risk factors include the postpartum state, adrenergic or serotonergic drugs, chronic migraines, and vasoactive material (cocaine, ecstasy, amphetamines, alcohol, and nicotine $)^{5}$. Post-partum RCVS usually occurs in the first week 

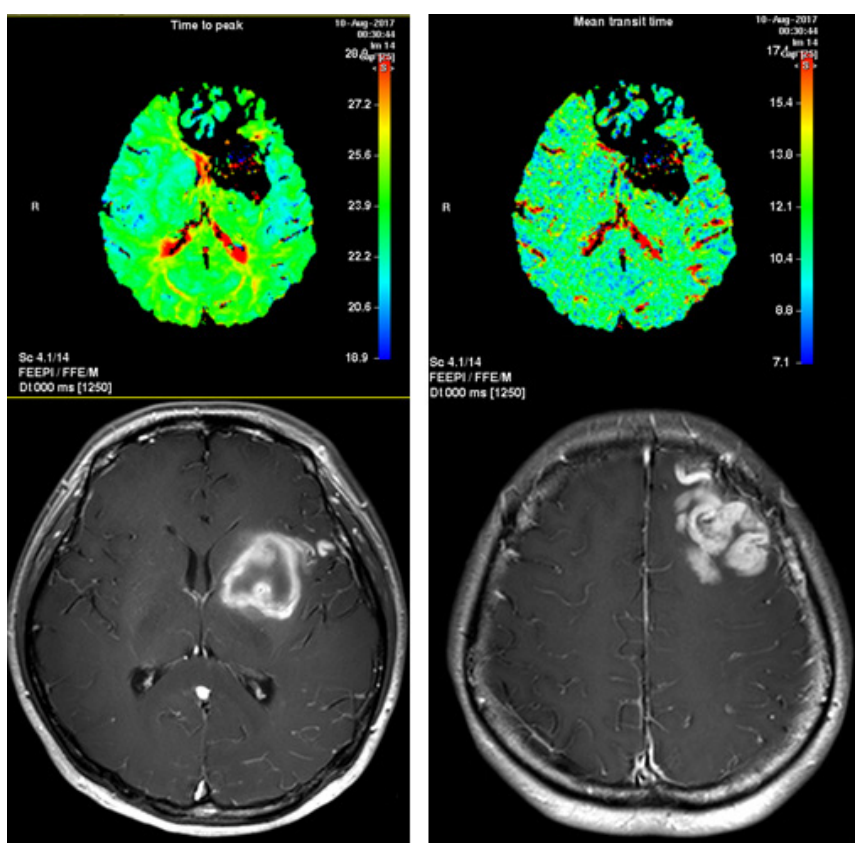

Fig. 5. Magnetic resonance (MR) perfusion on day 18. Time to peak and mean transit time delays were not found (upper). The MRI T1 contrast image revealed probable ICH in the Lt. basal ganglia (lower left) and gyral enhancement along the Lt. frontal convexity, with probable subacute infarction (lower right).
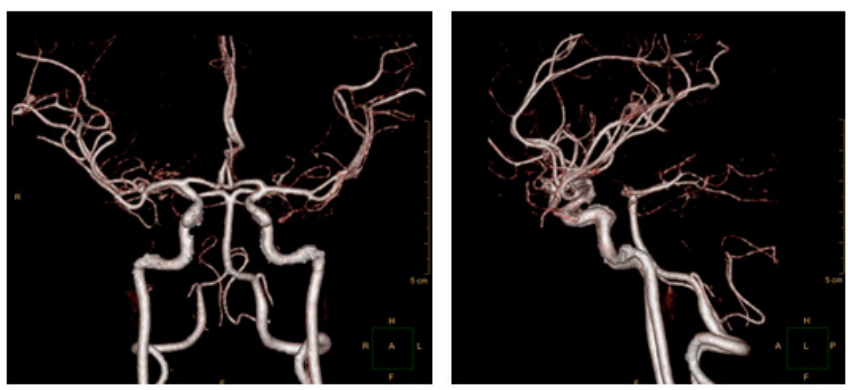

Fig. 6. CT angiography follow-up at three months. Diffuse vasospasm was not noted.

after delivery. Most cases are associated with administered vasoconstrictors, such as ergotamine and oxytocin ${ }^{5)}$.

RCVS can be differentially diagnosed by primary angiitis of the central nervous system (PACNS) and primary reversible cerebral vasoconstriction syndrome (PRES). Clinically, PACNS usually has a chronic onset, not significant thunderclap headache, and progresses to stepwise neurological deterioration. Because PACNS involves inflammation of the blood vessels, cerebrospinal fluid (CSF) analysis shows inflammatory changes. PACNS can be diagnosed by several occlusions seen on angiography, with similar angiographic findings on DSA and CTA. Mandell et al. reported that high-resolution magnetic resonance imaging (HRMRI) could be a diagnostic choice in differentiating the distinct wall characteristics of both diseases. The PACNS group showed wall thickening and wall enhancement, and the RCVS group exhibited wall thickening without wall enhancement. Obusez et al reported that HRMRI showed a continuous pattern in RCVS throughout the entire wall, with or without an enhancing wall pattern. This enhancing pattern is distinct in the short segmental concentric or eccentric enhancing wall pattern of PACNS ${ }^{5,6}$. With time, RCVS stabilizes, and the angiographic findings tend to improve quickly, whereas the arterial luminal irregularity in PACNS does not improve in a short time ${ }^{5)}$. Multiple cerebral infarctions can occur in PACNS and RCVS, and PACNS patients are typically prone to acute infarction in the border-zone territories ${ }^{7}$. In contrast, PACNS tends to cause acute infarction in the large branch artery territory or small vessel occlusion are ${ }^{8)}$. Intra-arterial vasodilators such as nimodipine have been proposed to have differential diagnostic value in RCVS. The drug immediately normalizes spasms in RCVS, whereas vasodilators infused at the lesion in PACNS do not consistently produce changes ${ }^{9}$. However, in our case, intra-arterial nimodipine infusion did not result in vasodilation. After nimodipine infusion, the overall arterial caliber was similar to that in the pre-treatment state.

RCVS and PRES overlap significantly in clinical and radiographic features. PRES has an acute onset, with severe headache, visual changes, and seizures, which can be reversible. MRI T2 images can differentiate PRES from RCVS, in which symmetric both side edema is seen in the cortex and subcortical white matter, mostly of the posterior parietal and occipital lobes, that quickly reverses. In PRES patients, on post-contrast images, superficial leptomeningeal enhancement is the most common finding. One-third of the patients exhibit combined gyral cortical enhancement in the T1 images $^{10-12)}$. Vasogenic edema occurs in the parietal-occipital regions in $90 \%$ of the patients ${ }^{12}$.

A quarter of the patients with PRES are normotensive. A more recent study reported that endothelial dysfunction could trigger the regulation of arterial tone and affect vasoconstriction with hypoperfusion ${ }^{13,14)}$. MR perfusion demonstrated reduced cortical relative cerebral blood volume(rCBV) in PRES lesions, and a stringof-beads pattern along with reduced $\mathrm{rCBV}$ indicates a hypoperfusion state in PRES $^{14)}$.

SAH or ICH in RCVS typically occurs in cerebral convexities a few days after the initial headache, before infarction ${ }^{15}$. Ducros et al. found different time courses of thunderclap headaches, vasoconstriction, and ischemia, suggesting that vasospasm starts in the small arterioles and gradually progresses toward medium-sized and large arteries ${ }^{15,16)}$. The mechanism of ICH and SAH in RCVS patients is still unknown. Recurrent vessel rupture and reperfusion injury from vasoconstriction and dilation of the leptomeningeal arteries is hypothesized ${ }^{16)}$. Endothelial dysfunction in vessel walls 
can be the key to explaining the stroke mechanism ${ }^{16)}$. The mechanism of intraventricular hemorrhage following RCVS has not been clearly defined. The blood supply of the fourth ventricle comes from the perforator branch of Posterior cerebral artery (PCAs) and superior cerebellar artery (SCAs) ${ }^{17}$. In our patient, (Intraventricular hemorrhage) IVH and left (basal ganglia) BG ICH was detected on the initial CT scan, supporting the theory that small arteries are more susceptible to repeated vasoconstriction and vasodilation than medium or large-sized arteries. Our findings support that these perforators may be affected by vasoconstriction and reperfusion.

From the treatment of RCVS, guidelines have been established by observational data and opinions ${ }^{18)}$. Because of the self-limiting disease entity, symptomatic treatment and Intensive care unit (ICU) administration following close blood pressure monitoring should be done. Vasoactive drugs should be avoided at first, and analgesic agents administered to relieve severe headaches and as seizure prophylaxis. Osmotic agents can also be used ${ }^{19)}$. Calcium channel blockers and magnesium sulfate have been reported to reduce vascular tone ${ }^{18,19)}$.

Long-term treatment should include the secondary prevention of stroke, and antihypertensive agents with calcium channel blockers and nimodipine can be considered. Oral or intravenous nimodipine has been reported to provide symptom relief, especially for headaches ${ }^{9}$. Balloon-angioplasty was reported in some cases, but four of five patients died ${ }^{20,21)}$. Intra-arterial vasodilator therapy has been reported to improve vasoconstriction. However, multiple treatments may be needed due to recurrent arterial spasms ${ }^{9,22)}$. In our case, intra-arterial nimodipine $3 \mathrm{mg}$ infusion was administered continuously for two days, yet the vasospasm did not improve (Fig. 4).

\section{CONCLUSION}

The pathophysiological mechanism of PACNS, PRES, and RCVS is still unknown. PACNS, PRES, and RCVS are clinically and radiologically similar with overlapping findings and are a part of a continuous disease entity, so the initial diagnosis can be difficult. Several theories have debated the involvement of cerebral autoregulation and endothelial dysfunction.

Developing MRI and neuroradiological studies and clinical findings may help with diagnosis and treatment. Discovering pathophysiologic algorithms and the accumulation of treated cases can improve clinical outcomes.

\section{NOTES}

\section{Conflict of interest}

No potential conflict of interest relevant to this article was reported.

\section{REFERENCES}

1. Topcuoglu MA, Singhal AB. Hemorrhagic reversible cerebral vasoconstriction syndrome: features and mechanisms. Stroke 2016;47:1742-1747.

2. Obusez E, Hui F, Hajj-Ali R, Cerejo R, Calabrese L, Hammad T, et al. High-resolution MRI vessel wall imaging: spatial and temporal patterns of reversible cerebral vasoconstriction syndrome and central nervous system vasculitis. AJNR Am J Neuroradiol 2014;35:1527-1532.

3. Choi K-S, Yi H-J. Progressive manifestations of reversible cerebral vasoconstriction syndrome presenting with subarachnoid hemorrhage, intracerebral hemorrhage, and cerebral infarction. J Korean Neurosurg Soc 2014;56:419.

4. Restrepo EQ Vélez SV, Herrera DA. Reversible cerebral vasoconstriction: subarachnoid convexity hemorrhage.

5. Hirunpat S, Sanghan N, Wan-ai A, Ina N, Hirunpat P. Reversible cerebral vasoconstriction syndrome: a commonly missed diagnosis in acute severe headaches. ASEAN J Radiol 2013;19: 154-163.

6. Mandell DM, Matouk CC, Farb RI, Krings T, Agid R, TerBrugge $\mathrm{K}$, et al. Vessel wall MRI to differentiate between reversible cerebral vasoconstriction syndrome and central nervous system vasculitis: preliminary results. Stroke 2012;43:860-2.

7. Ducros A. Reversible cerebral vasoconstriction syndrome. The Lancet Neurology 2012;11:906-917.

8. Hajj-Ali RA, Singhal AB, Benseler S, Molloy E, Calabrese LH. Primary angiitis of the CNS. The Lancet Neurology 2011;10: 561-572.

9. Elstner M, Linn J, Müller-Schunk S, Straube A. Reversible cerebral vasoconstriction syndrome: a complicated clinical course treated with intra-arterial application of nimodipine. Cephalalgia 2009;29:677-682.

10. Levitt A, Zampolin R, Burns J, Bello JA, Slasky SE. Posterior reversible encephalopathy syndrome and reversible cerebral vasoconstriction syndrome: distinct clinical entities with overlapping pathophysiology. Radiologic Clinics 2019;57:1133-1146.

11. Miller T, Shivashankar R, Mossa-Basha M, Gandhi D. Reversible cerebral vasoconstriction syndrome, part 1: epidemiology, pathogenesis, and clinical course. AJNR Am J Neuroradiol 2015;36:1392-9. 
12. Pilato F, Distefano M, Calandrelli R. Posterior reversible encephalopathy syndrome and reversible cerebral vasoconstriction syndrome: clinical and radiological considerations. Front neurol 2020;11:34

13. Edlow BL, Kasner SE, Hurst RW, Weigele JB, Levine JM. Reversible cerebral vasoconstriction syndrome associated with subarachnoid hemorrhage. Neurocrit Care 2007;7:203-210.

14. Bartynski W, Boardman J. Catheter angiography, MR angiography, and MR perfusion in posterior reversible encephalopathy syndrome. AJNR Am J Neuroradiol 2008;29:447-45.

15. Ducros A, Fiedler U, Porcher R, Boukobza M, Stapf C, Bousser M-G. Hemorrhagic manifestations of reversible cerebral vasoconstriction syndrome: frequency, features, and risk factors. Stroke 2010;41:2505-2511.

16. Ducros A, Boukobza M, Porcher R, Sarov M, Valade D, Bousser $\mathrm{M}-\mathrm{G}$. The clinical and radiological spectrum of reversible cerebral vasoconstriction syndrome. A prospective series of $67 \mathrm{pa}-$ tients. Brain 2007;130:3091-3101.

17. Sharifi M, Ciołkowski M, Krajewski P, Ciszek B. The choroid plexus of the fourth ventricle and its arteries. Folia Morphol (Warsz) 2005;64:194-198.

18. Calabrese LH, Dodick DW, Schwedt TJ, Singhal AB. Narrative review: reversible cerebral vasoconstriction syndromes. Ann Intern Med 2007;146:34-44.

19. Ducros A. Reversible cerebral vasoconstriction syndrome. Handbook of clinical neurology 2014;121:1725-1741.

20. Fugate JE, Wijdicks EF, Parisi JE, Kallmes DF, Cloft HJ, Flemming KD, et al. Fulminant postpartum cerebral vasoconstriction syndrome. Arch Neurol 2012;69:111-117.

21. Song J, Fisher S, Seifert T, Cacayorin E, Alexandrov A, Malkoff $\mathrm{M}$, et al. Postpartum cerebral angiopathy: atypical features and treatment with intracranial balloon angioplasty. Neuroradiology 2004;46:1022-1026.

22. French KF, Hoesch RE, Allred J, Wilder M, Smith A, Digre KB, et al. Repetitive use of intra-arterial verapamil in the treatment of reversible cerebral vasoconstriction syndrome. J Clin Neurosci 2012;19:174-176. 